\title{
Die Anfänge des Frauenstudiums und ein Blick in die Praxis
}

\section{Verena E. Müller}

lic. phil., Historikerin, Zürich

\author{
Voraussetzung für eine Pioniertat sind Begabung, Durchhaltewillen, der richtige \\ Zeitpunkt, ein unterstützendes Umfeld und viel Glück. Im Leben der ersten Schwei- \\ zer Ärztin scheinen diese Bedingungen optimal erfüllt. Ohne das Einverständnis \\ und den gewaltigen materiellen Einsatz ihres Vaters Julius Vögtlin wäre sie wohl \\ wie ihre ältere Schwester Anna eine ehrbare alte Jungfer in Brugg geworden. Prakti- \\ zieren konnte sie nur mit der Erlaubnis ihres Gatten Albert Heim. Dass sie für zahl- \\ reiche junge Frauen zum Vorbild wurde, ist jedoch ganz ihr Verdienst.
}

\begin{abstract}
Niemand hätte der Pfarrerstochter Marie Vögtlin bei ihrer Geburt in Bözen AG am 7. Oktober 1845 eine aussergewöhnliche Zukunft vorausgesagt. Ihre Biographie verlief in üblichen Bahnen: Unterricht zu Hause statt in der überfüllten Dorfschule, Besuch des Neuenburger Töchterpensionats Montmirail, Erlernen der Führung eines gepflegten Haushalts bei Cousine Blumer in Zürich, Verlobung mit Cousin Friedrich Erismann (1842-1915), der Lebensweg einer Arztgattin schien vorgezeichnet.

Dank der Begegnung mit Friedrich Erismann kam Marie Vögtlin in Berührung mit anderen Lebensmustern und neuen Ideen. Dieser Cousin, auch er ein Pfarrerssohn, war früh Vollwaise, studierte Medizin und wurde Augenarzt. Als elternloser Student hatte der unruhige Geist mehr Freiheiten als seine Kommilitonen und wusste diese zu nützen. In Zürich traf er Nadezhda Suslova, jene russische Studentin, die als erste ein Medizinstudium abschloss. Seine Verlobung mit Marie löste
\end{abstract}
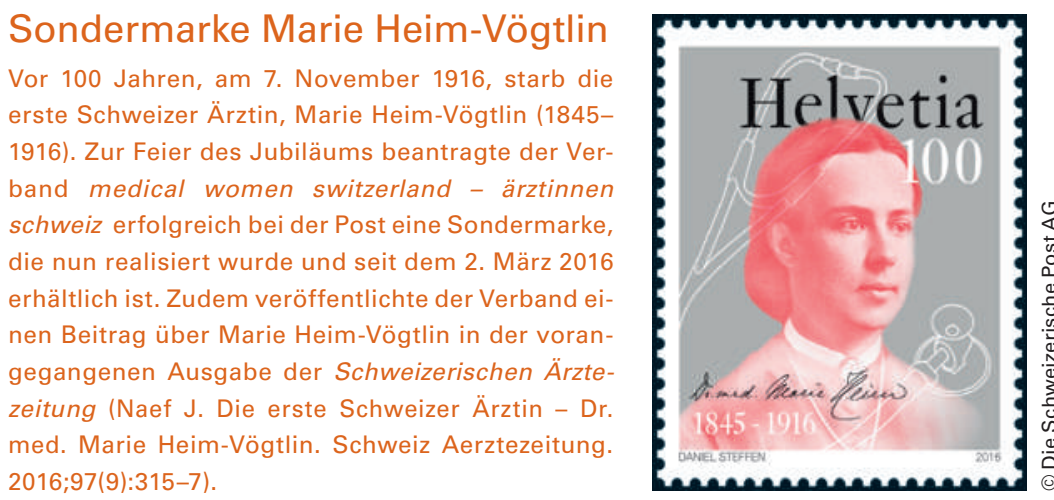

er auf, heiratete die Berufskollegin und zog mit ihr nach St. Petersburg. Allerdings scheiterte die Ehe nach einigen Jahren. Erismann wurde Professor für Hygiene in Mos-

\section{Der Lebensweg einer Arztgattin schien vorgezeichnet.}

kau und gründete mit einer anderen ehemaligen Zürcher Studentin, Sophie Hasse, eine Familie. Mit der Zeit missfielen Erismanns progressive Ideen dem Zaren. Nach der unfreiwilligen Rückkehr in die Schweiz beendete er seine Karriere als Vertreter der Sozialdemokraten im Zürcher Stadtrat (Exekutive).

Die Trennung von Erismann warf Marie Vögtlin aus der Bahn. Sie erkrankte. Der schwere Liebeskummer löste jedoch eine unerwartete Entwicklung aus. Nun ohne Aussicht auf Heirat und Kinder, entschloss sie sich, selber Ärztin zu werden. Zwar verfügte sie über solide Fremdsprachenkenntnisse, doch fehlte ihr mathematisches und naturwissenschaftliches Grundlagenwissen für das Medizinstudium. Da die Universität nur von Zürcher Bürgern ein Maturitätszeugnis verlangte, konnten die ersten Studentinnen - aus dem Aargau oder aus dem Ausland - ihr Studium auf Zusehen hin aufnehmen.

Die meisten Professoren der Medizinischen Fakultät standen dem Frauenstudium pragmatisch-wohlwollend gegenüber. Dies ist umso bemerkenswerter, als einige von ihnen jung genug waren, um auf die Fortsetzung ihrer Karriere an renommierteren Hochschulen zu hoffen, die dem Frauenstudium kritisch gegenüber standen. Wäre das Experiment gescheitert, hätte dies ihre eigenen beruflichen Aussichten beeinträchtigt. 
Ein weiterer Glücksfall: Nadezhda Suslova war intelligent, fleissig und fiel nie unangenehm auf. Damit machte sie den Weg für ihre Nachfolgerinnen frei.

Die Zürcher Studenten benahmen sich neugierig-höflich und unterschieden sich damit positiv von jungen Männern an gewissen ausländischen Universitäten. Als diese Institutionen Frauen endlich zur Ausbildung zuliessen, verhielten sich die Herren Kommilitonen oftmals rüpelhaft. Anders in Zürich: Einige Studenten verliebten sich gar mehr oder weniger erfolgreich in eine Kollegin. - Auguste Forel (1848-1931), bekannter Ameisenforscher und Psychiater, ist ein ergiebiger Zeitzeuge.

\section{Die meisten Professoren der Medizinischen Fakultät standen dem Frauenstudium pragmatisch-wohlwollend gegenüber.}

Wie Marie Heim-Vögtlin wurde er später zum Vorkämpfer der Abstinenz. In seinen Briefen an die Eltern bestellte er nicht nur ein weiteres Fässchen Wein, sondern berichtete der Mutter ausführlich vom Tun und Lassen der Kommilitoninnen, die auf Ausflügen brav Milch tranken, wie er ausdrücklich vermerkte. Marie Vögtlins Briefe schildern fröhliche Expeditionen. Sie beklagte sich, dass an Regensonntagen keine botanischen Ausflüge stattfanden, weil die Herren «zu bequem zum Marschieren» seien. Bei Exkursionen, die Übernachtungen voraussetzten, kam eine neutrale Begleitung mit, etwa der Pfarrer von Wiedikon, um den guten Ruf der jungen Frauen nicht zu gefährden. Marie Vögtlin und ihre Studienfreundin Susan Dimock waren ausgezeichnete Bergsteigerinnen. Wahrscheinlich war auch diese Sportlichkeit hilfreich im Umgang mit den Kollegen.

Hätte Marie Vögtlin das Studium ohne die treue Unterstützung durch ihren Freundeskreis geschafft? Ihre geheimen Studienpläne besprach sie zuerst mit Marie Ritter (1842-1933), einer Pfarrerstochter aus Schwanden GL. Grossmütig ermunterte diese Marie Vögtlin, den ehrgeizigen Plan zu verfolgen, obschon sie selber - zwar hoch begabt - keine beruflichen Perspektiven hatte. Erst nach dieser Ermutigung wagte es Marie Vögtlin, ihrem Vater das Projekt vorzulegen. - Gleich zu Beginn des Studiums begegnete sie der ersten amerikanischen Studentin in Zürich, Susan Dimock (18471875) aus Boston. Nachdem ihr die Harvard Medical School den Zutritt verweigert hatte, finanzierten Gönnerinnen ihren Aufenthalt sowie die sehr hohen Studiengebühren (die HMS liess sich Zeit, Frauen nahm sie erst ab 1945 auf). Vögtlin und Dimock bildeten ein Team, Marie erteilte der Freundin Deutschunterricht, Susan gab Nachhilfe in den Naturwissenschaften, denn sie hatte bereits als Praktikantin in einem Frauenspital gearbeitet.

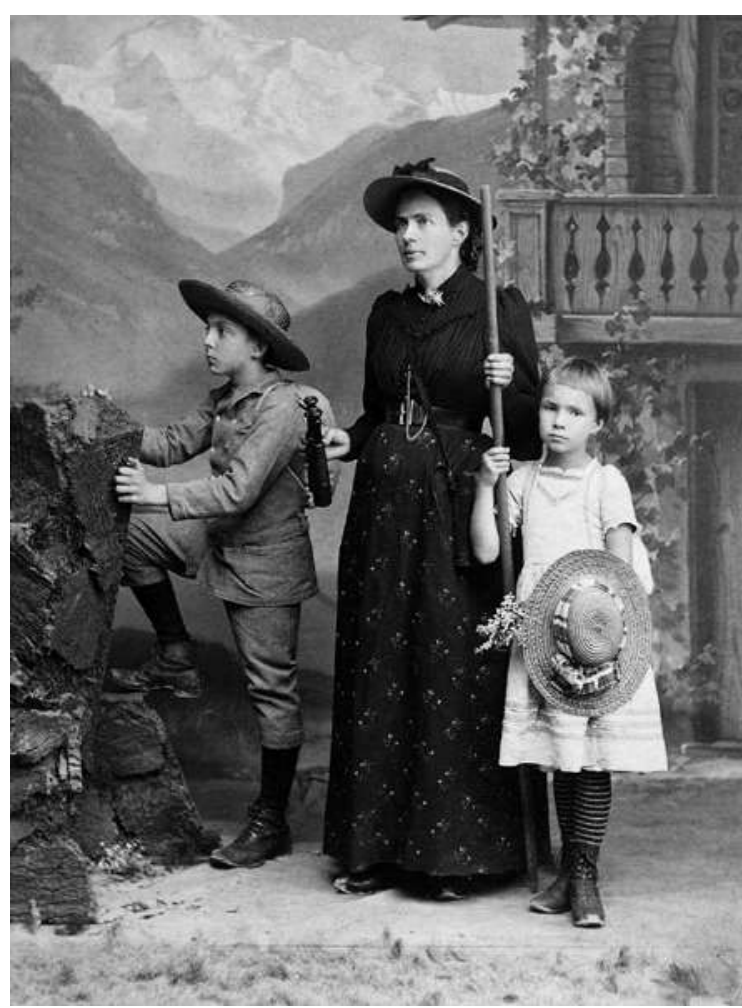

Marie Heim-Vögtlin war eine begeisterte Bergsteigerin. Hier zu sehen mit Sohn Arnold und Tochter Helene.

Im Hause ihrer Cousine Rudolphine Blumer lernte Marie Vögtlin die Italienischlehrerin Sophie Heim (1847-1931) kennen. Rasch entwickelte sich eine enge Freundschaft. Familie Heim nahm die Studentinnen Vögtlin und Dimock mit offenen Armen auf. Man las gemeinsam Bücher, diskutierte, machte Hausmusik. Während einiger Zeit lebte Susan Dimock bei Familie Heim sogar als Untermieterin; Marie Vögtlin verlobte sich mit dem Sohn des Hauses, Albert (1849-1937). Zu den glücklichen Fügungen zählt die Beziehung zur künftigen Schwiegerfamilie. Hier fühlte sich Marie Vögtlin im wahrsten Sinne des Wortes ganz und gar «heimisch». Nach ihrem Zürcher Abschluss verbrachte Susan Dimock gleichzeitig mit Forel ein Kliniksemester in Wien.

\section{Familie Heim nahm die Studentinnen Vögtlin und Dimock mit offenen Armen auf.}

Sie kehrte in die USA zurück und arbeitete wie vorgesehen im New England Hospital for Women and Children. 1875 hatte sie die Stipendien zurückbezahlt und plante eine Reise nach Europa. Gross war im Freundeskreis die Bestürzung, als die «Schiller» vor der Küste Cornwalls in Seenot geriet und Susan Dimock ertrank. George Hoggan, der Ehemann ihrer einstigen Zürcher Studienkollegin Frances Morgan (1843-1927), identifizierte die Tote. Tief betrübt beschrieb Forel das Unglück 
seiner Mutter, und Gustav Adolf Tobler (1850-1923), später Professor für Schwachstromtechnik an der ETH, erkannte, dass seine Liebe zu Susan für immer einseitig bleiben würde.

Kaum hatte Marie Vögtlin 1874 die Doktorprüfung bestanden, eröffnete sie ihre Praxis in Hottingen, damals ein selbständiges Dorf vor den Toren der Stadt Zürich. Heute erinnert eine Tafel an der Hottingerstrasse 25 an die Pionierin. Über ihren beruflichen Alltag wissen wir wenig. Der Tagesablauf war streng geregelt, denn neben ihrem Beruf trug sie die Verantwortung für das Hauswesen, unterstützt selbstverständlich von entsprechendem Personal. Diese Hilfen waren nicht immer so tüchtig wie die erste Magd Henriette Schaffner, die Marie Heim-Vögtlin von ihrer Tante Erismann «geerbt» hatte.

Sprechstunde hielt Marie Heim-Vögtlin am Nachmittag. Hausbesuche oder die umfangreiche Korrespondenz erledigte sie am Vormittag oder abends. Regelmässig holten sich auswärtige Patientinnen schriftlich bei ihr Rat. Auch hier ist nur ein Beispiel überliefert. Nach-

\section{Kaum hatte Marie Vögtlin 1874 die Doktor- prüfung bestanden, eröffnete sie ihre Praxis in Hottingen.}

dem die Ärztin 1887 die in Basel an einer Unterleibsinfektion erkrankte Gattin von Albert Heims Jugendfreund Eugen Huber besucht hatte, betreute sie die Patientin aus der Ferne. In seinem Tagebuch notierte Huber «... ihre Briefe über die mögliche Aetiologie von

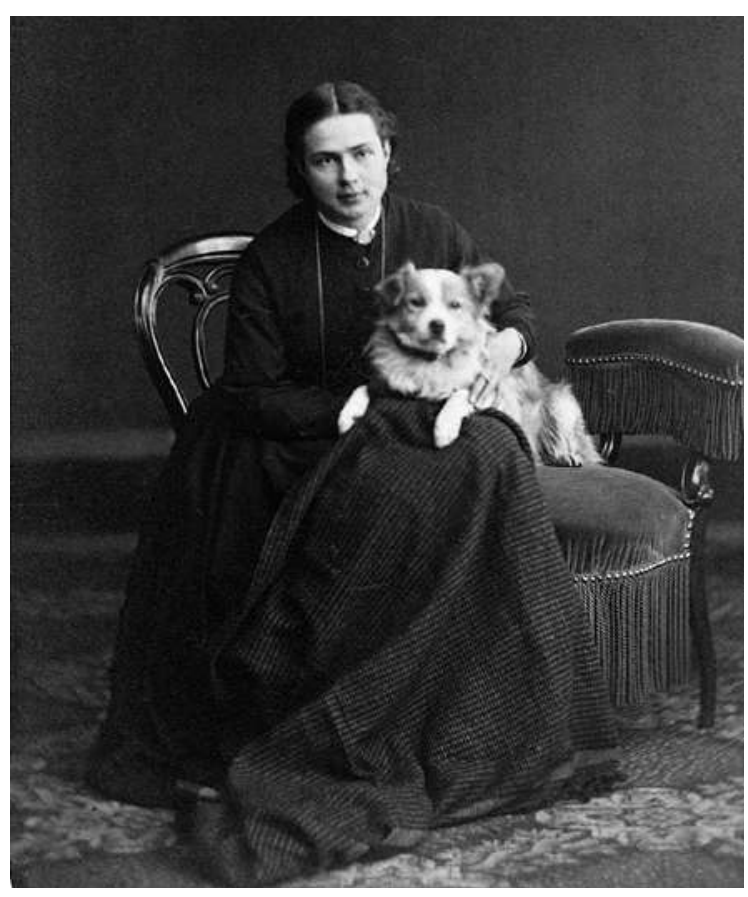

Marie Heim-Vögtlin mit Hund Leo.
Linas Krankheit. Ich weiss, was ich zu thun habe.» $\mathrm{Zu}$ Hubers grossem Missfallen hatte ihn Marie HeimVögtlin aus dem ehelichen Schlafzimmer verbannt. Um Lina zu schonen, musste er in einer andern Kammer übernachten.

Administratives war schon damals ein Zeitfresser. Rechnungen stellte die Ärztin zwar nur aufs Jahresende und jeweils über zwölf Monate, bei «armen Wiibli» soll sie diese bereits quittiert verschickt haben. - Häufig wurde

\section{Marie Heim-Vögtlin war eine Praktikerin,} die ungern wissenschaftliche Artikel verfasste.

Marie Heim-Vögtlin in der Nacht zu Geburten gerufen. Solche Einsätze bezahlte sie oft mit quälenden Kopfschmerzen oder tagelangen Migräneanfällen, Erholung fand sie in der Gartenarbeit.

Marie Heim-Vögtlin war eine Praktikerin, die ungern wissenschaftliche Artikel verfasste. Einzig 1879 veröffentlichte sie im «Correspondenz-Blatt» der Schweizer Ärzte einen längeren Bericht über die Behandlung von Blasenerkrankungen. In einem der Fälle hatte die $\mathrm{Pa}$ tientin seit 27, in einem anderen seit 23 Jahren ununterbrochen an Blutungen gelitten. Aus Scham hatten diese Frauen offenbar keinen Arzt aufgesucht, ihnen brachte sie Heilung. «Die simonschen Harnröhrendilatiationen liessen sich in tiefer Narkose durchführen", beschrieb sie den Eingriff. Diese frühen Operationen führte sie in Anwesenheit eines Kollegen in der eigenen Praxis durch. Sie war vorsichtig genug, zu Beginn ihrer Tätigkeit ihren Ruf nicht aufs Spiel zu setzen.

Bei bösartigen Tumoren konnte auch Marie HeimVögtlin nichts ausrichten, die Betroffenen starben, wie sie im Artikel schrieb. Dies galt auch noch Jahrzehnte später. Eine der wenigen namentlich bekannten $\mathrm{Pa}$ tientinnen war die Schriftstellerin Johanna Spyri (18271901), eine Jugendfreundin von Albert Heims Tante, der Kunstmalerin Nannette Fries. Die Ärztin begleitete die Krebskranke bis zum Tod.

Drei Jahrzehnte nach Marie Heim-Vögtlins Doktorat veröffentlichte ihr ehemaliger Verlobter Erismann eine Abhandlung zum Frauenstudium. «Die Furcht vor dem Untergang des 'ewig Weiblichen' bei akademisch gebildeten Frauen erwies sich als grundlos und Haus und Familie hatten nicht nur keine Verluste zu beklagen, sondern konnten sich ökonomisch und kulturell ganz entschiedener Gewinne rühmen.» Er musste es wissen, war er doch mit drei Pionierinnen bestens bekannt.

Bildnachweis

Marie Heim-Vögtlin mit Familie und Hund Privatarchiv Verena E. Müller 